\title{
KDM6B Elicits Cell Apoptosis by Promoting Nuclear Translocation of FOXO1 in Non- Small Cell Lung Cancer
}

\author{
Jun Ma ${ }^{a}$ Ning Wang ${ }^{a}$ Yurong Zhang ${ }^{a}$ Cun Wang ${ }^{a}$ Tianxiang Ge \\ Haojie Jin ${ }^{a}$ Xuan Denga Xisong Huo ${ }^{a}$ Dishui Gua Zhouhong Ge ${ }^{a}$ Wei Chu \\ Liyan Jiang ${ }^{\mathrm{b}}$ Wenxin Qin ${ }^{\mathrm{a}}$
}

\begin{abstract}
aState Key Laboratory of Oncogenes and Related Genes, Shanghai Cancer Institute, Renji Hospital, Shanghai Jiao Tong University School of Medicine, Shanghai, bDepartment of Pulmonary Medicine, Shanghai Chest Hospital, Shanghai Jiao Tong University, Shanghai, China
\end{abstract}

\author{
Key Words \\ KDM6B • Nonsmall cell lung cancer • Apoptosis • FOXO1 • Metastasis
}

\begin{abstract}
Background/Aims: Non-small cell lung carcinoma (NSCLC) is the most common type of lung cancer and the cause of most cancer-related deaths. The molecular mechanisms that are involved in NSCLC development are currently not well understood. Accumulating evidence shows that histone demethylases play important roles in the regulation of pathological developmental processes in many diseases, including various types of cancers. Methods: Mitochondrial membrane potential assays, migration and invasion assays, caspase-3 and caspase- 9 activity assays and western blot analysis were used in this research. Results: We found that overexpression of KDM6B, a demethylase that acts on histone H3 at lysine 27 (H3K27), inhibited cell growth by initiating mitochondria-dependent apoptosis and by attenuating the invasion-metastasis cascade in NSCLC cells. Moreover, our results showed that KDM6B directly interacted with FOXO1 and that overexpression of KDM6B promoted nuclear accumulation of FOXO1. The effects of KDM6B on cell apoptosis and metastasis were weakened by knockdown of FOXO1 expression. On the contrary, knocking down expression of KDM6B inhibited cell apoptosis and promoted cell growth by mitigating the nuclear translocation of FOXO1 in NSCLC cells. Conclusions: These findings suggest that KDM6B may act in a pro-apoptotic role in NSCLC by causing the nuclear translocation of FOXO1.

Copyright $\odot 2015$ S. Karger AG, Basel
\end{abstract}

\section{Introduction}

Lung cancer is one of the most common malignancies and the leading cause of cancerrelated deaths in the world $[1,2]$. Non-small cell lung carcinoma (NSCLC) is the most common 


\section{Cellular Physiology Cell Physiol Biochem 2015;37:201-213 \\ \begin{tabular}{l|l}
\hline DOI: 10.1159/000430345 & (C) 2015 S. Karger AG, Basel
\end{tabular} \\ and Biochemistry Published online: August 20, $2015 \quad$ www.karger.com/cpb \\ Ma et al.: KDM6B Elicits Apoptosis by FOXO1 in NSCLCS}

histological type of lung cancer and accounts for more than $85 \%$ of the cases [3]. The main types of NSCLC are adenocarcinoma and squamous cell carcinoma [4]. Although some advanced treatment techniques have been developed, the 5-year survival rate of patients with NSCLC remains poor [5]. Moreover, the molecular mechanisms involved in NSCLC are poorly understood. Therefore, it is necessary to identify novel genes that participate in regulating the progression and development of NSCLC.

The development of tumors often results from an imbalance between cell proliferation and apoptosis. Apoptosis plays a negative regulatory role that acts against the initiation and progression of cancer. Genes associated with the regulation of apoptosis are considered to be a new class of tumor-related genes [6]. Histone methylation, which is regulated by histone methyltransferases and demethylases, participates in the regulation of transcriptional repression and activation during both normal embryonic development and carcinogenic progression [7, 8]. Multiple cellular functions, including cell proliferation, apoptosis and differentiation, are affected by histone methylation. Previous studies have shown that some histone methyltransferases and demethylases are closely related to cell growth, metastasis and apoptosis during the progression and development of cancer [9-11].

The trimethylation of lysine 27 of histone $\mathrm{H} 3$ (H3K27me3) is an important function of histone modification. Accumulating evidence has shown that KDM6B (lysine (K)-specific demethylase 6B), an $\mathrm{H} 3 \mathrm{~K} 27$ demethylase, is a crucial regulator of the progression of tumorigenesis. However, the reported roles played by KDM6B in different human cancers are contradictory. It has been reported, for example, that KDM6B inhibits tumor progression in both pancreatic and colon cancer $[12,13]$. It has also been reported that KDM6B, serving as an oncogene, enhances tumorigenesis and promotes the development of tumors in patients with Hodgkin's lymphoma, breast carcinoma, and renal cell carcinoma [14-16]. Currently, there is no direct evidence regarding the effect of KDM6B on the progression of NSCLC. Therefore, a potential role for KDM6B in apoptosis in NSCLC cells remains unknown.

In this study, we first found that overexpression of KDM6B elicited mitochondriadependent apoptosis and significantly inhibited cell growth in NSCLC cells. Knockdown of KDM6B facilitated cell growth and inhibited cell apoptosis. The underlying molecular mechanisms involving KDM6B in apoptosis were also explored.

\section{Materials and Methods}

\section{Cell culture}

The human NSCLC cell lines A549, H460, H1299, SK-MES-1, H1650, H292, and H358 were purchased from American Tissue Culture Collection (ATCC). All cells were cultured in Dulbecco's modified Eagle's medium (DMEM) (Gibco, CA, USA), with 10\% fetal bovine serum (FBS) in a $5 \% \mathrm{CO}_{2}$ atmosphere at $37^{\circ} \mathrm{C}$.

\section{Western blot assay}

Cell proteins were extracted with the T-PER tissue protein extraction reagent (Pierce, Rockford, IL) containing the appropriate concentrations of proteinase inhibitors and phosphatase inhibitors (Roche Applied Science). The modified Bradford assay (Sigma) was used for detecting the total protein concentration. The protein samples were fractionated by SDS-PAGE (12\% polyacrylamide gels) and transferred onto nitrocellulose membranes (Bio-Rad, Hercules, CA). After incubation in a blocking buffer (Tris $20 \mathrm{mM}, \mathrm{pH}$ 7.6, NaCl $150 \mathrm{mM}$, and Tween 20 0.1\%) containing 5\% nonfat dry milk powder, the membranes were incubated with FOX01 (1:1000 dilution; Cell Signaling Technology), p-FOX01 (1:1000 dilution; Cell Signaling Technology), Bim (1:1000 dilution; Cell Signaling Technology), KDM6B (1:1000 dilution; Abcam, Hong Kong, China) or beta-actin (1:5000 dilution; Kang-Chen, Shanghai, China) overnight at $4^{\circ} \mathrm{C}$, followed by reaction with HRP-conjugated secondary antibodies for $1 \mathrm{~h}$ at room temperature and enhanced chemiluminescence reagents, protein bands were visualized using chemiluminescence detection. Beta-actin was utilized as an internal control.

\section{KARGER}




\section{Cellular Physiology Cell Physiol Biochem 2015;37:201-213 \begin{tabular}{l|l} 
DOI: 10.1159/000430345 & O 2015 S. Karger AG, Basel
\end{tabular} and Biochemistry Published online: August 20, 2015 www.karger.com/cpb \\ Ma et al.: KDM6B Elicits Apoptosis by FOXO1 in NSCLCS}

RNA isolation and real-time RT-PCR

Total RNA was extracted from cells using TRIzol reagent (Invitrogen). The PrimeScript ${ }^{\mathrm{TM}}$ RT Reagent Kit (TaKaRa Biotechnology) was utilized for reverse transcription. Real-time polymerase chain reaction (PCR) was subsequently performed according to the manufacturer's instructions (Takara Biotechnology). Primers for target genes were designed and are listed as follows: KDM6B forward (5'-3'): GGT TCC AAT GAG ACA GGGCA, reverse (5'-3'): CAG GAC TTC CTC TCT CCCCA; beta-actin forward (5'-3'): TTG TTA CAG GAA GTC CCT TGCC, reverse (5'-3'): ATG CTA TCA CCT CCC CTG TGTG. Expression levels were normalized against the internal reference gene beta-actin and represented using the $2^{-\Delta \Delta C t}$ method.

\section{Lentivirus production and cell transduction}

The cDNA encoding KDM6B (NM_001080424) was amplified by reverse transcription polymerase chain reaction (RT-PCR). The cDNA of KDM6B was cloned into the pGC-FU vector. Lentiviruses carrying KDM6B cDNA or KDM6B shRNA were generated and harvested according to the manufacturer's instruction. The shRNA with a non-targeting sequence was used as a negative control. Briefly, target cells $\left(1 \times 10^{5}\right)$, including A549, H460, H1299 and H1650, were infected with $1 \times 10^{6}$ recombinant lentivirus-transducing units in the presence of $6 \mu \mathrm{g} / \mathrm{ml}$ polybrene (Sigma). The NSCLC cells that were stably infected with recombinant lentivirus were named A549 KDM6B (overexpression) and A549 GFP (control) and H460 KDM6B (overexpression) and H460 GFP (control), H1299 shKDM6B (knockdown) and H1299 shControl (control), and H1650 shKDM6B (knockdown) and H1650 shControl (control).

\section{MTT assay}

Cells were cultured at a density of 5,000 cells/well in a 96-well culture plate. After $24 \mathrm{~h}$, cells were incubated for $4 \mathrm{~h}$ in medium containing 0.5\% 3-[4, 5-dimethylthiazol-2-yl]-2,5-diphenyltetrazolium bromide (MTT), which is a yellow mitochondrial dye. The reaction was terminated with $150 \mu \mathrm{l}$ of DMSO for $10 \mathrm{~min}$. Absorbance was read at $540 \mathrm{~nm}$ using a microplate reader. The experiment was performed in triplicate.

\section{FOXO1 knockdown with short interfering RNA}

Short interfering RNA (siRNA) directed specifically against FOXO1 (Biotend, Shanghai, China) was transfected into NSCLCs using Lipofectamine 2000 reagent (Invitrogen, Carlsbad, CA, USA) according to the manufacturer's instructions. The siRNA sequence targeting human FOXO1 was: 5' -GGAGAAGAGCUGCAUCCAUTT-3'. The knockdown efficiency of the FOXO1 siRNA was analyzed by western blot.

\section{$L D H$ release assay}

Activity of lactate dehydrogenase (LDH) released into culture media was determined using a cytotoxicity detection kit. Briefly, a portion of culture medium was reacted with an equal volume of LDH substrate solution for $30 \mathrm{~min}$. The reaction was stopped by adding 5 volumes of $0.1 \mathrm{M} \mathrm{NaOH}$, and the absorbance was then measured at $440 \mathrm{~nm}$ using a spectrophotometer. Sister cultures were treated with a $1 / 100$ volume of $10 \%$ Triton X-100 and incubated at $37^{\circ} \mathrm{C}$ for $30 \mathrm{~min}$. Total LDH activity was determined using medium containing Triton-lysed cellular supernatant.

\section{Migration and invasion assays}

Cell migration and invasion assays were performed using a 24-well transwell plate (BD Biosciences, CA, USA). For the transwell migration assay, $3 \times 10^{4} \mathrm{~A} 549$ and $5 \times 10^{4} \mathrm{H} 460$ cells were resuspended in $0.2 \mathrm{ml}$ of DMEM and seeded in the upper chamber of a transwell. For the transwell invasion assays, $4 \times 10^{4} \mathrm{~A} 549$ and $6 \times 10^{4} \mathrm{H} 460$ cells were resuspended in $0.2 \mathrm{ml}$ of DMEM and seeded in Matrigel (BD Biosciences, CA, USA)-coated chamber inserts. A volume of $0.8 \mathrm{ml}$ DMEM containing 10\% FBS was then placed in the lower chamber. After an optimal incubation time, we used a cotton swab to remove non-migrated and non-invaded cells from the upper chamber. The migrated or invaded cells were stained with crystal violet $(1 \%$ in methyl alcohol) for $10 \mathrm{~min}$.

Mitochondrial membrane potentials assay

Mitochondrial depolarization in A549 and H460 cells was examined using a JC-1 probe (Beyotime, Nantong, China). Cells were cultured at a density of $5 \times 10^{4} / \mathrm{ml}$ in six-well plates according to the 


\section{Cellular Physiology Cell Physiol Biochem 2015;37:201-213 \\ \begin{tabular}{l|l|l}
\cline { 2 - 3 } DOI: 10.1159/000430345 & (C) 2015 S. Karger AG, Basel
\end{tabular} \\ and Biochemistry Published online: August 20, $2015 \quad$ www.karger.com/cpb \\ Ma et al.: KDM6B Elicits Apoptosis by FOXO1 in NSCLCs}

manufacturer's instructions. After $24 \mathrm{~h}$, all cells were resuspended and collected, and the probe JC-1 was added to cells for $20 \mathrm{~min}$ at $37^{\circ} \mathrm{C}$. After rinsing cells twice with PBS, fluorescence was immediately analyzed by a flow cytometer (Becton Dickinson). The relative amounts of dual emissions from JC-1 monomers or aggregates were used to reflect the mitochondrial membrane potentials.

\section{Hoechst staining}

Cells were cultured in six-well chamber slides to $\sim 60 \%$ confluency. One microliter of Hoechst 33342 ( $5 \mathrm{mg} / \mathrm{ml}$, Sigma) was diluted with $1 \mathrm{ml}$ of basal medium and cells were stained with the diluents and incubated for $30 \mathrm{~min}$. Stained cells were washed twice with cold PBS and imaged under a fluorescent microscope using 350-nm stimulation and 460-nm emission.

Measurement of caspase- 3 and caspase- 9 activity

Levels of caspase- 3 and caspase- 9 activity were measured by cleavage of a caspase- 3 substrate, AcDEVD-pNA (acetyl-Asp-Glu-Val-Asp p-nitroanilide), and cleavage of a caspase-9 substrate, Ac-LEHD-pNA (acetyl-Leu-Glu-His-Asp p-nitroanilide), respectively. Briefly, protein samples were extracted as described for western blot analysis. Approximately $50 \mathrm{mg}$ of total protein was then added to a reaction buffer containing Ac-DEVD-pNA ( $2 \mathrm{mM}$ ) or Ac-LEHD-pNA $(2 \mathrm{mM})$ for $2 \mathrm{~h}$ at $37^{\circ} \mathrm{C}$. The absorbance of the substrates was measured by a spectrometer at $405 \mathrm{~nm}$. The specific activity levels of caspase- 3 and caspase- 9 were then normalized to the total protein of respective cell lysates.

\section{Statistical analysis}

Data are expressed as the mean \pm standard error of the mean (SEM) from at least three independent experiments. Statistical analysis was performed using a chi-square test, student's t-test or one-way ANOVA followed by Dunnett's test where appropriate. $\mathrm{p}<0.05$ was considered statistically significant.

\section{Results}

Expression analysis of KDM6B in NSCLC cell lines

We first examined the expression of KDM6B in seven NSCLC cells lines. As shown in Fig. 1A, real-time PCR was used to confirm the mRNA expression of KDM6B. MTT results showed that cells with lower expression of KDM6B generally grew faster in the seven NSCLC cells lines (Fig. 1B, n=3, p<0.05). As for the growth rate of H1299, a possible explanation is because p53 is completely absent in H1299, which needs to be verified in further studies.

Moreover, we found that expression of KDM6B was lower in the A549 and H460 cell lines compared to other cell lines. Thus, we established the steady overexpression of KDM6B in A549 and H460. Based on real-time PCR and western blot analysis, KDM6B was stably over-expressed in both cell lines (Fig. 1C and 1D, n=3, p<0.05).

Overexpression of KDM6B inhibits cell growth by cell apoptosis

MTT and LDH assays were used to explore the role of KDM6B in the growth of NSCLC cells. We found that cell viability was significantly decreased by KDM6B overexpression (Fig. $1 \mathrm{E}, \mathrm{n}=3, \mathrm{p}<0.05$ ). Cell death experiments showed that KDM6B overexpression increased the release of LDH in A549 and H460 cells (Fig. 1F, n=3, p<0.05). These results indicate that KDM6B inhibits growth and causes cell death in NSCLC cells.

Apoptosis is an important process that regulates cell growth. Therefore, we explored whether the inhibitory effects of KDM6B on cell growth are accomplished through an increase in cell apoptosis. As shown in Fig. 1G, overexpression of KDM6B triggered the activation of caspase-3 in A549 and H460 cells. Apoptosis-related changes to nuclear morphology were also observed by staining with Hoechst 33342. The proportion of abnormal nuclear characteristics (including crenation, condensation and fragmentation) was substantially increased by KDM6B overexpression (Fig. $1 \mathrm{H}, \mathrm{n}=3, \mathrm{p}<0.05$ ). These results suggest that KDM6B likely has a pro-apoptotic function in NSCLC. 


\section{Cellular Physiology $\frac{\text { Cell Physiol Biochem 2015;37:201-213 }}{\text { DOI: 10.1159/000430345 }}$ \begin{tabular}{ll|l}
\cline { 2 - 2 } and Biochemistry $10.1159 / 000430345$ & O 2015 S. Karger AG, Base \\
\hline
\end{tabular}

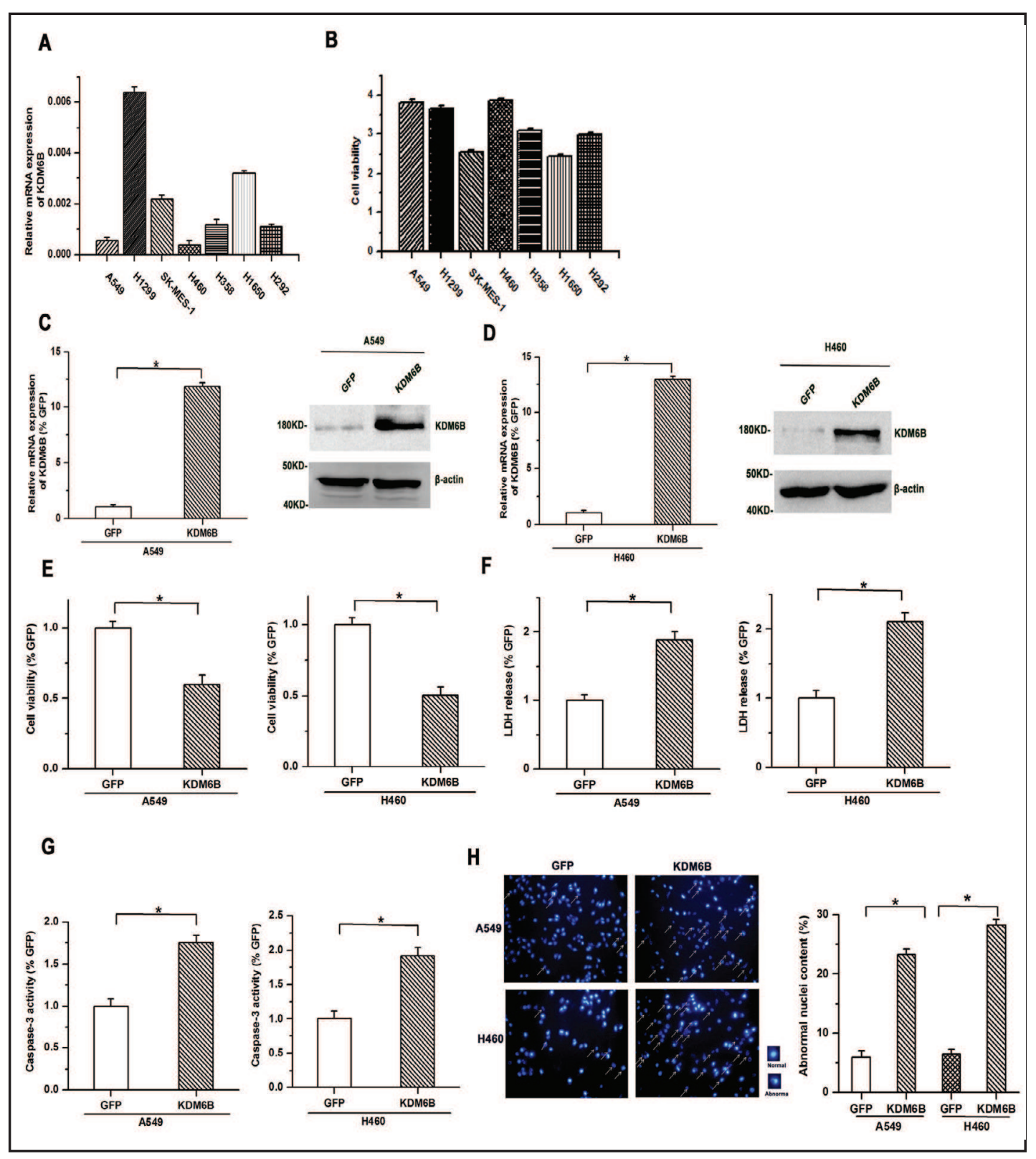

Fig. 1. Overexpression of KDM6B inhibits growth by promoting cell apoptosis in A549 and H460 cells. A: mRNA level of KDM6B was examined in seven NSCLC cell lines by real-time PCR. B: MTT results showed that cells with lower expression of KDM6B generally grew faster in seven NSCLC cells lines. C and D: Overexpression of KDM6B in A549 and H460 cells that were stably infected with KDM6B-expressing lentivirus or controls was verified by real-time PCR and western blot assay, respectively. E: Cell viability was significantly decreased by KDM6B overexpression. F: Release of LDH was induced in A549 and H460 cells by overexpression of KDM6B. G: Overexpression of KDM6B promoted the activation of caspase- 3 and potentiated the activity of caspase-3 in A549 and H460 cells. H: The proportion of abnormal nuclear characteristics was increased by KDM6B overexpression.

Mitochondria-dependent apoptosis is initiated by KDM6B overexpression

Intrinsic cell apoptosis is usually initiated by a decrease in mitochondrial membrane potential (MMP). To further investigate the role of KDM6B in cell apoptosis, the probe JC-1 was used to determine the effects of KDM6B on changes to MMP. As shown in Fig. 2A, flow cytometry assays revealed that the KDM6B overexpression in A549 cells resulted in an 


\section{\begin{tabular}{ll|l}
\cline { 3 - 3 } Cellular Physiology & \multicolumn{3}{l}{ Cell Physiol Biochem 2015;37:201-213 } & \\
\cline { 3 - 3 } DOI: 10.1159/000430345 & O 2015 S. Karger AG, Basel
\end{tabular} \begin{tabular}{l|l} 
and Biochemistry Published online: August 20, 2015 & $\begin{array}{l}\text { O 2015 S. Karger AG, Buser } \\
\text { www.karger.com/cpb }\end{array}$ \\
\cline { 1 - 2 } &
\end{tabular}}

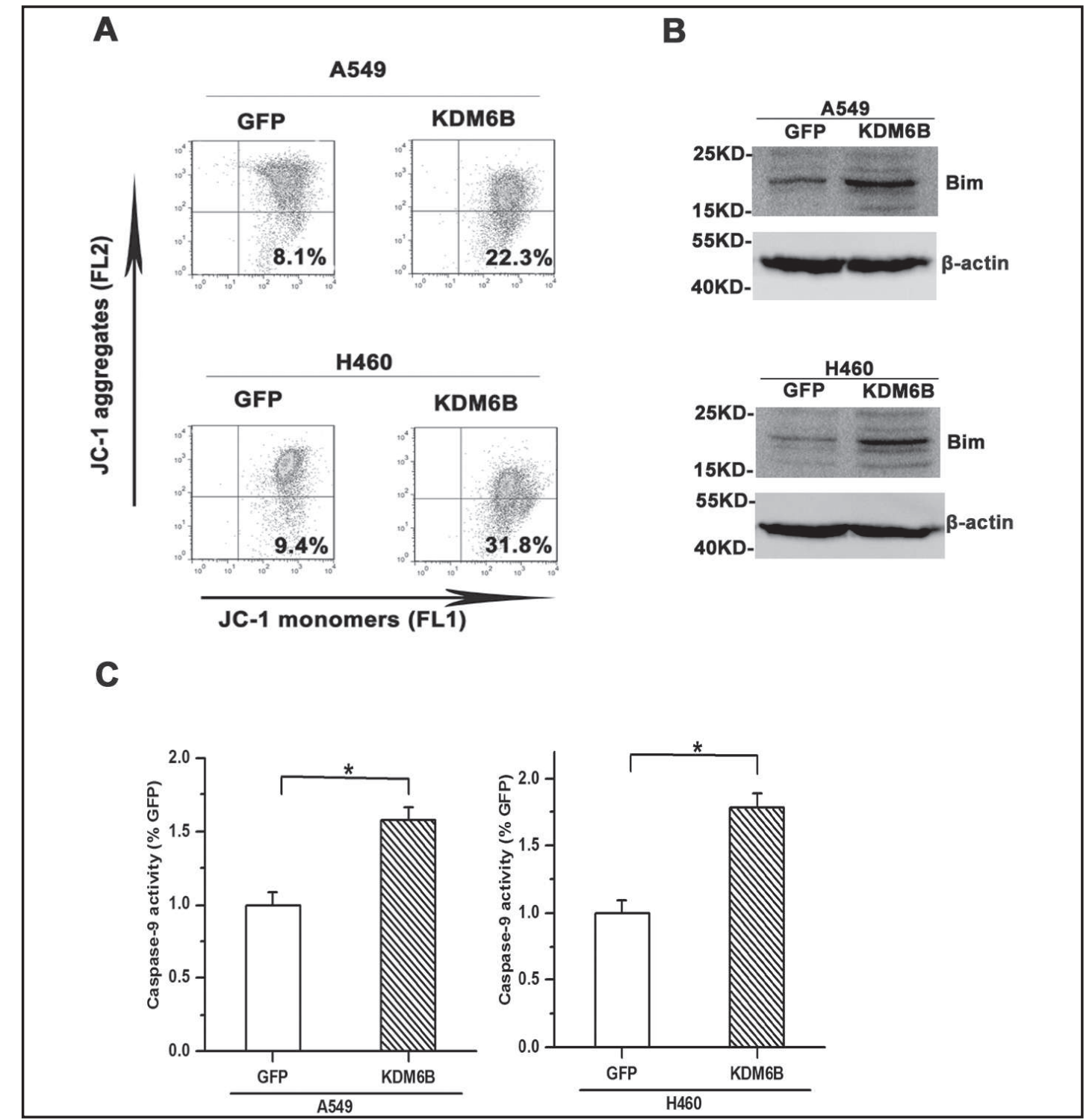

$\mathbf{B}$

Fig. 2. Mitochondria-dependent apoptosis is initiated by KDM6B overexpression. A: Flow cytometry assay showed that KDM6B overexpression led to a decrease in MMP and disrupted mitochondrial integrity in A549 and H460 cells. B: Overexpression of KDM6B increased expression of Bim. C: Overexpression of KDM6B resulted in increased caspase- 9 activity in A549 and H460 cells. Data are presented as the mean \pm SEM (for each group $\mathrm{n}=3 ;{ }^{*} \mathrm{p}<0.05$ ).

increase in the number of cells with low MMP, from $8.1 \%$ to $22.3 \%$. Similar results were observed in $\mathrm{H} 460$ cells, where KDM6B overexpression increased the number of cells with low MMP, from $9.4 \%$ to $31.8 \%$ (Fig. $2 \mathrm{~A}, \mathrm{p}<0.05$ ). The above results suggest that KDM6B overexpression induces a decrease in MMP by disrupting mitochondrial integrity.

Bim also plays a key role in regulating MMP. Increased expression of Bim leads to a decline in MMP and triggers mitochondria-dependent apoptosis. We next studied whether Bim was affected by KDM6B overexpression. We observed that KDM6B overexpression significantly elevated the expression of Bim (Fig. 2B, n=3, p $<0.05$ ). Caspase-9, which is specifically activated in mitochondria-dependent apoptosis, was also examined. We found that overexpression of KDM6B in A549 and H460 cells increased caspase-9 activity (Fig. 2C, $\mathrm{n}=3, \mathrm{p}<0.05$ ). These results show that mitochondria-dependent apoptosis is increased by KDM6B overexpression in NSCLC. 


\section{Cellular Physiology Cell Physiol Biochem 2015;37:201-213 \begin{tabular}{l|l|l} 
DOI: 10.1159/000430345 & O 2015 S. Karger AG, Basel
\end{tabular} and Biochemistry Published online: August 20, 2015 www.karger.com/cpb

Fig. 3. KDM6B interacts with FOX01 and promotes the nuclear accumulation of FOXO1. A: KDM6B overexpression decreased phosphorylation of FOXO1 in whole cell lysates. B: KDM6B overexpression led to a significant increase in nuclear accumulation of FOXO1 in A549 and H460 cells. C: Overexpression of KDM6B enhanced endogenous interactions between F0X01 and KDM6B. All experiments are performed in triplicate and represented as the mean \pm SEM $\left({ }^{*} \mathrm{p}<0.05\right)$.

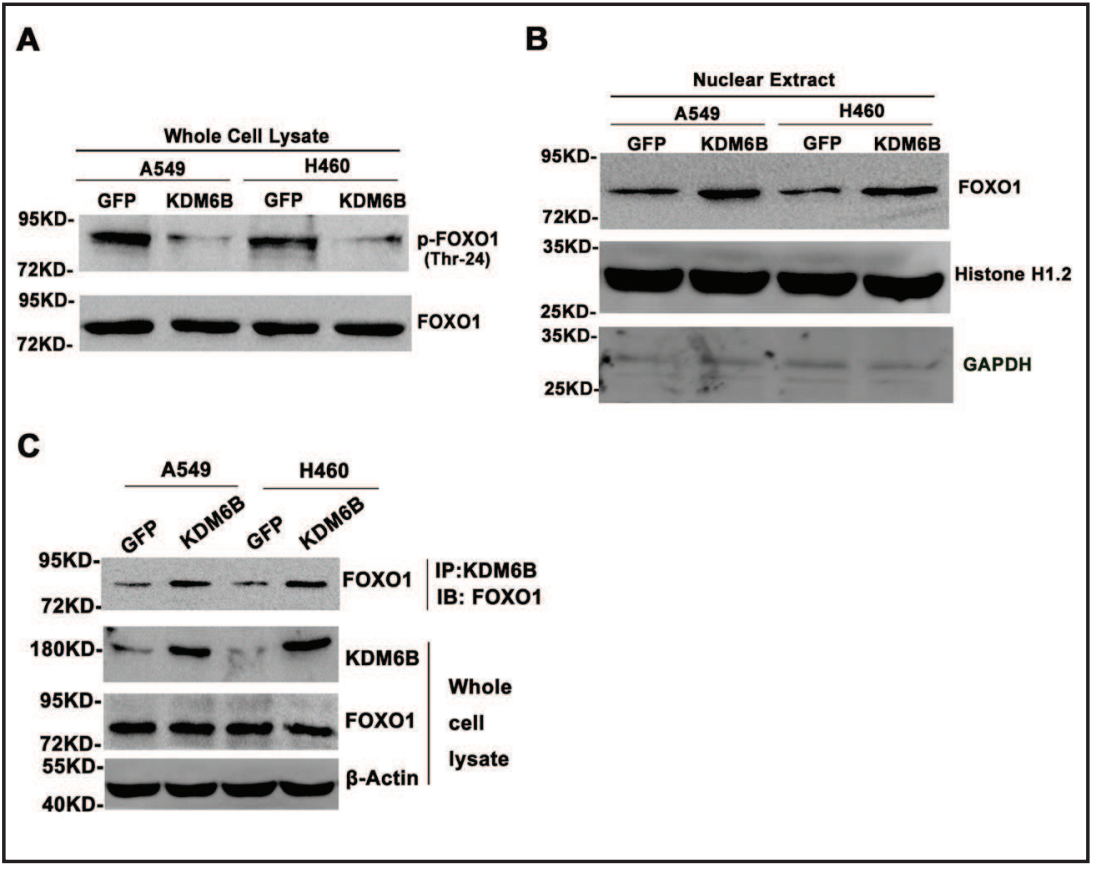

KDM6B interacts with FOXO1 and promotes its nuclear accumulation

FOX01 has been reported to play a key role in inducing apoptosis by targeting Bim, a transcription factor, and by using KDM6B to execute its function through interactions with other proteins in the cell nucleus [17-19]. To study this potential mechanism of KDM6Bfacilitated cell apoptosis, we explored whether there was a link between KDM6B and FOXO1. As shown in Fig. 3A and 3B, KDM6B overexpression decreased phosphorylation of FOXO1 in whole cell lysates and increased the accumulation of FOXO1 in nuclear lysates in A549 and $\mathrm{H} 460$ cells. Moreover, co-immunoprecipitation results showed that overexpression of KDM6B enhanced endogenous interactions between FOXO1 and KDM6B in A549 and H460 cells (Fig. $3 \mathrm{C}, \mathrm{n}=3, \mathrm{p}<0.05$ ). These results collectively indicate that KDM6B directly binds to FOXO1 and increases FOXO1 accumulation in the nucleus.

Pro-apoptotic effects of KDM6B are weakened by FOXO1 knockdown

Because we found that KDM6B interacts directly with FOXO1 and promotes its nuclear accumulation, we next used siRNA targeting FOXO1 to test whether FOXO1 participates in KDM6B-facilitated apoptosis. The efficiency of FOXO1 knockdown by FOXO1 siRNA (siFOXO1) in A549 and H460 was examined by western blot analysis (Fig. $4 \mathrm{~A}, \mathrm{n}=3, \mathrm{p}<0.05$ ). As shown in Fig. 4B, KDM6B-induced inhibition of cell viability was reversed by siFOX01. Knockdown of FOXO1 also decreased KDM6B-induced activation of caspase-3 (Fig. 4C, n=3, p<0.05). Moreover, KDM6B-induced increases in the expression of Bim and activated caspase- 9 were also reduced by siFOXO1 (Fig. 4D and $4 \mathrm{E}, \mathrm{n}=3, \mathrm{p}<0.05$ ). These results suggest that KDM6Binduced apoptosis is mediated by FOXO1.

FOXO1 mediates the inhibition of cell apoptosis induced by knockdown of KDM6B

To represent a physiological function of KDM6B, we also knocked down KDM6B gene expression in $\mathrm{H} 1299$ and H1650. The efficiency of knockdown was confirmed by real-time PCR and western blot (Fig. 5A and 5B, n=3, p<0.05). As shown in Fig. 5C and 5D, knocking down expression of KDM6B led to the increase of FOX01 phosphorylation in whole cell lysates and depressed the accumulation of FOXO1 in nuclear lysates in H1299 and H1650 cells. Furthermore, wortmannin $(500 \mathrm{nM})$, an inhibitor of the up-stream regulator of F0X01, was able to inhibit the phosphorylation of FOX01 (Fig. 5E, n=3, p<0.05). We found knockdown of KDM6B potentiated cell viability and inhibited activation of caspase-3, which 


\section{Cellular Physiology $\frac{\text { Cell Physiol Biochem 2015;37:201-213 }}{\text { DOI: 10.1159/000430345 }}$ \begin{tabular}{ll|l} 
DOI: 10.1159/000430345 & & \\
and Biochemistry & 2015 S. Karger AG, Bas
\end{tabular}

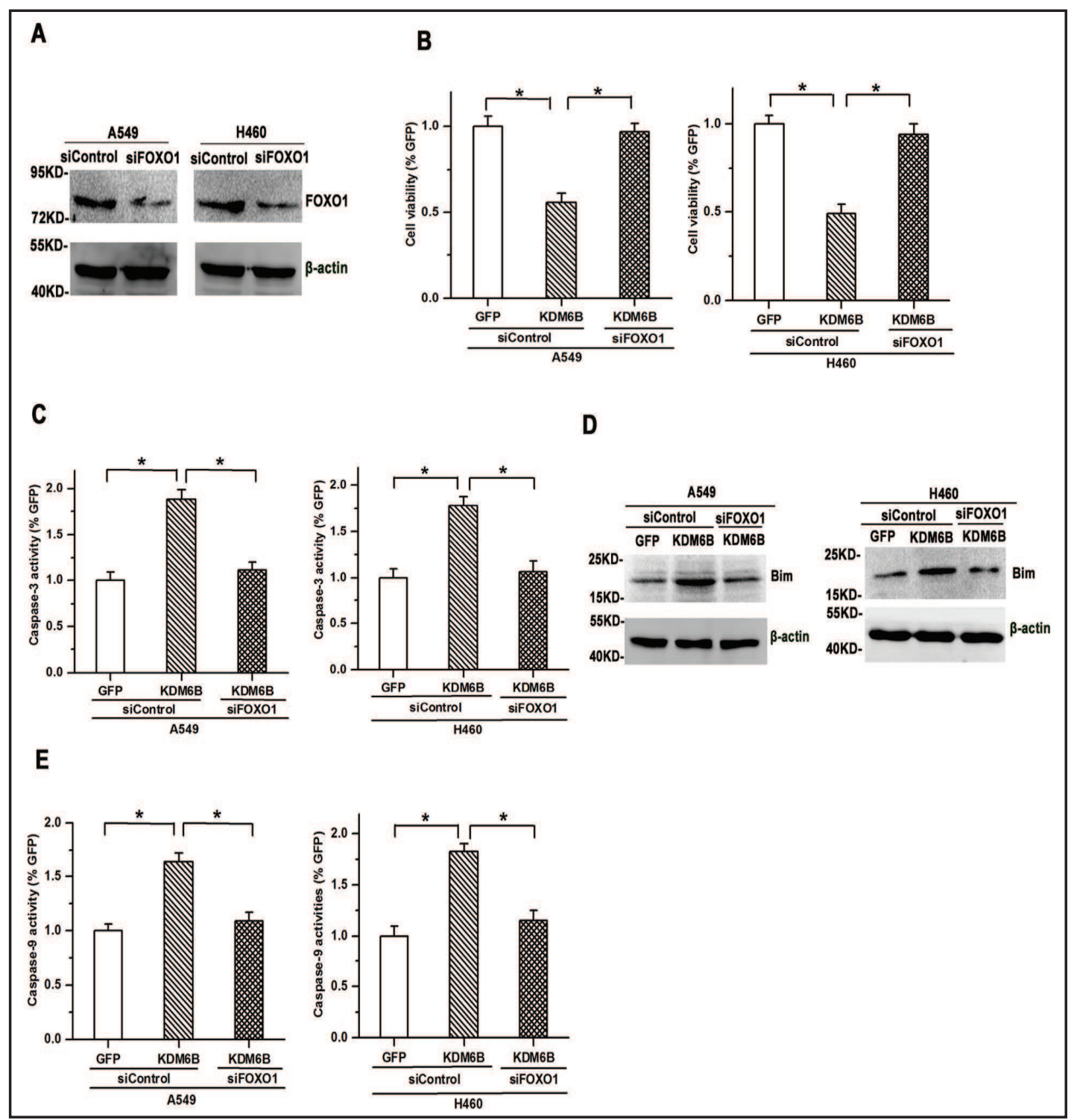

Fig. 4. The pro-apoptotic effects of KDM6B are weakened by knockdown of FOXO1 expression. A: Efficiency of FOXO1 knockdown by siFOXO1 was confirmed by western blot in A549 and H460 cells. B: The KDM6B-induced decrease in cell viability was repressed by siF0X01. C: Knockdown of F0X01 expression also depressed KDM6B-induced activation of caspase-3. D: The KDM6B-induced increase in expression of Bim was reduced by siFOXO1. E: FOX01 siRNA mitigated KDM6B-induced activation of caspase-9.

was significantly reversed by depressing FOXO1 phosphorylation with wortmannin (Fig. 5F and $5 \mathrm{G}, \mathrm{n}=3, \mathrm{p}<0.05)$. The inhibitory effects of KDM6B knockdown on Bim expression and caspase- 9 activation were blocked by wortmannin (Fig. $5 \mathrm{H}$ and $5 \mathrm{I}, \mathrm{n}=3, \mathrm{p}<0.05$ ). The results indicate that knockdown of KDM6B represses cell apoptosis and promotes cell growth by mitigating the nuclear translocation of FOXO1.

\section{KDM6B inhibits invasion-metastasis cascades in NSCLC by targeting FOXO1}

Invasion-metastasis cascades, which can result in the dissemination of cancer cells to anatomically distant organs, are important events in the development of cancers. To explore the role of KDM6B in the progression of NSCLC, we evaluated whether KDM6B is involved in migration and invasion in NSCLC. Overexpression of KDM6B significantly reduced the 


\section{Cellular Physiology Cell Physiol Biochem 2015;37:201-213 \begin{tabular}{l|l} 
DOI: 10.1159/000430345 & O 2015 S. Karger AG, Basel
\end{tabular} and Biochemistry Published online: August 20, $2015 \quad$ www.karger.com/cpb

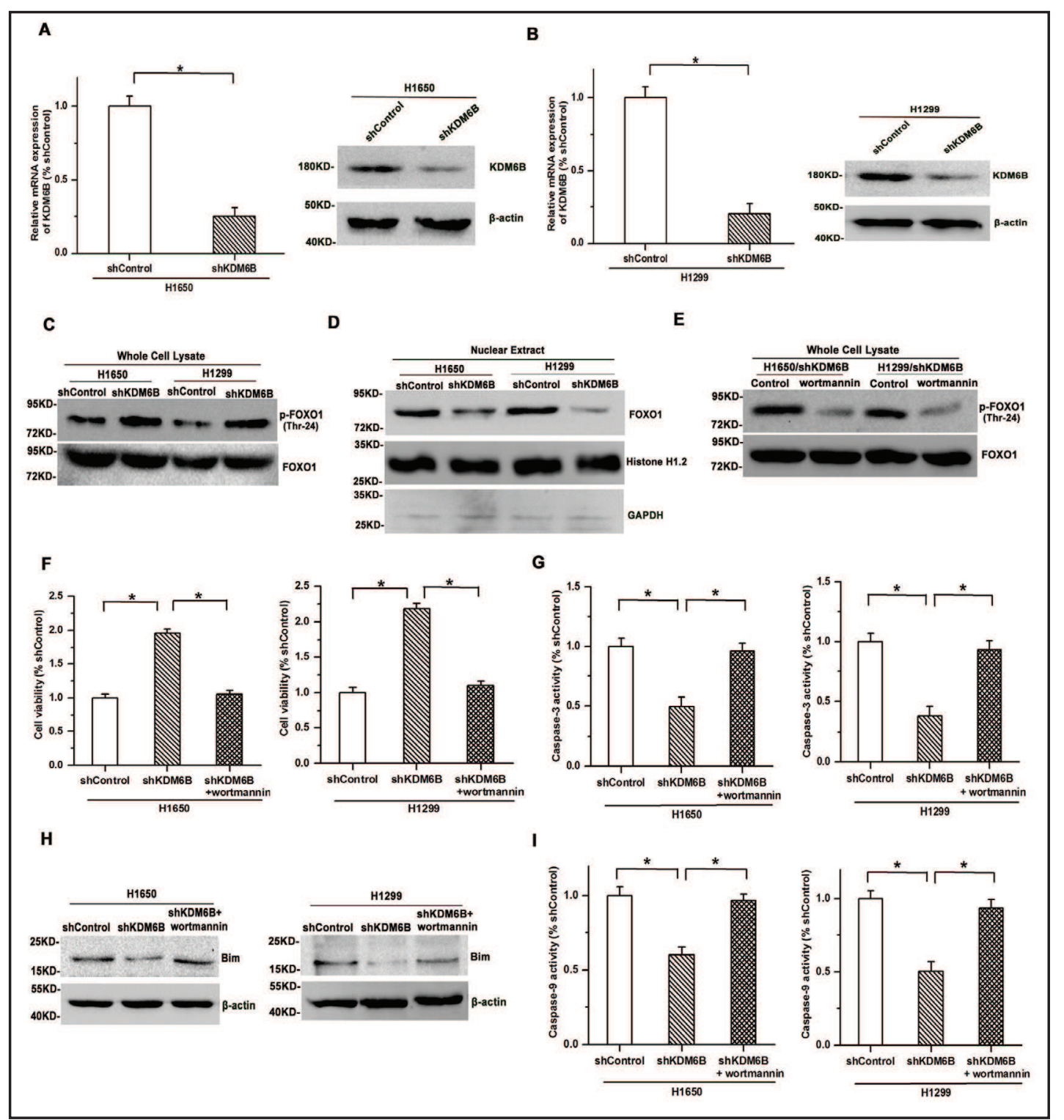

Fig. 5. Knockdown of KDM6B-induced inhibition of cell apoptosis is mediated by FOX01. A and B: Real-time PCR and western blot were used to confirm the efficiency of knockdown. C: knockdown of KDM6B led to the increase of FOXO1 phosphorylation in whole cell lysates. D: Accumulation of FOXO1 in nuclear lysates was repressed by knockdown of KDM6B in H1299 and H1650 cells. E: Phosphorylation of FOXO1 induced by KDM6B knockdown was mitigated by wortmannin. F: Knocking down expression of KDM6B potentiated cell viability in H1299 and H1650 cells, which was reversed by wortmannin. G: KDM6B knockdown-inhibited activation of caspase-3 was mediated by FOXO1. H: The inhibitory effects of KDM6B knockdown on Bim expression were blocked by wortmannin. I: KDM6B knockdown-inhibited activation of caspase-9 was mediated by FOXO1.

migratory capabilities of A549 and H460 cells. KDM6B overexpression also mitigated the invasive behavior of A549 and H460 cells. Treatment with siFOXO1 blocked the effect of KDM6B overexpression on both migration and invasion in these cell lines (Fig. 6A-6D, $\mathrm{n}=3, \mathrm{p}<0.05$ ). On the contrary, knocking down expression of KDM6B obviously enhanced the migration and invasive behavior of H1299 and H1650 cells, while the effects of KDM6B knockdown on metastasis were antagonized by wortmannin (Fig. 6E-6H, n=3, p<0.05). These results show that KDM6B inhibits migration and invasion via a mechanism involving FOXO1 in NSCLC. 


\section{Cellular Physiology Cell Physiol Biochem 2015;37:201-213 \begin{tabular}{l|l|l|l|l|l}
\hline DOI: 10.1159/000430345 & O 2015 S. Karger AG, Basel
\end{tabular} and Biochemistry Published online: August 20, $2015 \quad$ www.karger.com/cpb}

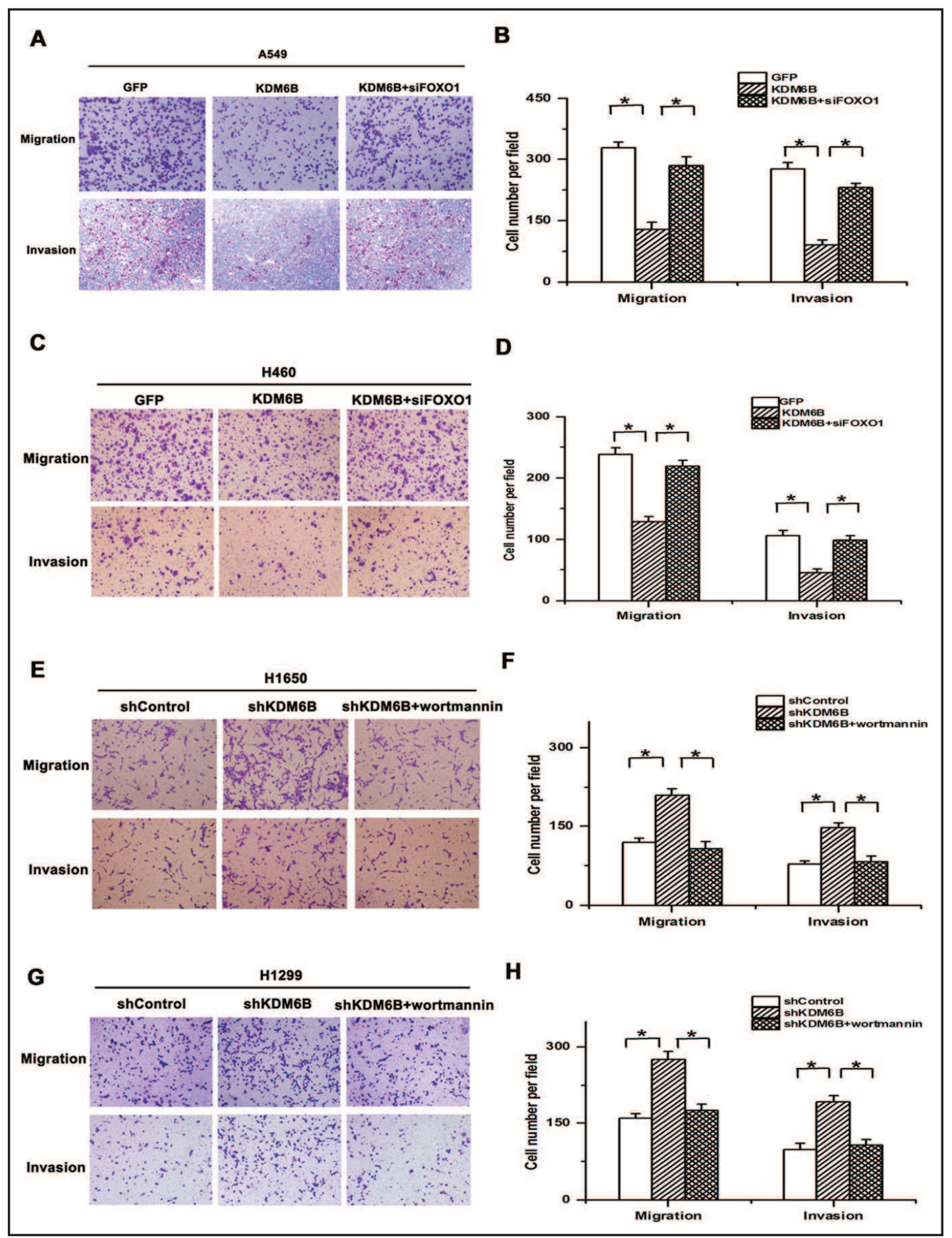

Fig. 6. KDM6B inhibits invasion-metastasis cascades in NSCLC by targeting FOXO1. A: Overexpression of KDM6B attenuated migration and invasion in A549 cells; these effects were eliminated by siF0X01. B: Quantitative analysis of cell numbers in migration and invasion assays. C: Overexpression of KDM6B attenuated migration and invasion through an interaction with FOXO1 in H460 cells. D: Quantitative analysis of cell numbers in migration and invasion assays. E and F: Knocking down expression of KDM6B obviously enhanced the migration and invasive behavior of $\mathrm{H} 1650$ cells, which was antagonized by wortmannin. $\mathrm{G}$ and H: Promotive effects of KDM6B knockdown on migration and invasion were mediated by FOXO1 in H1299 cells. Cell numbers in migration and invasion assays were calculated microscopically and are represented as the mean $\pm \mathrm{SEM}$. 


\section{Cellular Physiology Cell Physiol Biochem 2015;37:201-213 \\ \begin{tabular}{ll|l} 
and Biochemistry & $\begin{array}{l}\text { DOI: 10.1159/000430345 } \\
\text { Published online: August 20, } 2015\end{array}$ & $\begin{array}{l}\text { (c) } 2015 \mathrm{~S} \text {. Karger AG, Basel } \\
\text { www.karger.com/cpb }\end{array}$ \\
\cline { 2 - 3 } Ma et al: KDM6B Elicits Apoptosis by FOXO1 in NSCLCs
\end{tabular}}

\section{Discussion}

Mounting evidence indicates that KDM6B participates in the regulation of cancer development. The effects of KDM6B on tumorigenesis are not, however, consistent across different human cancers [12-16]. Knockdown of KDM6B has been shown to promote tumor sphere formation and increase peritoneal dissemination and liver metastasis in pancreatic adenocarcinoma [12]. The aggressiveness of colon cancer cells and the epithelial-to-mesenchymal transition are also enhanced by KDM6B knockdown [13]. However, KDM6B expression is significantly increased in Hodgkin's lymphoma and renal cell carcinoma compared to adjacent normal tissue $[14,16]$ and overexpression of KDM6B has been shown to induce the expression of mesenchymal genes and to promote invasionmetastasis cascades in breast carcinoma [15]. The specific role of KDM6B in NSCLC cells has therefore remained uncertain. In the present study, we provide new evidence showing that KDM6B induces mitochondria-dependent apoptosis by promoting the nuclear translocation of FOXO1 in NSCLC. To the best of our knowledge, this is the first study to demonstrate a proapoptotic role for KDM6B in NSCLC.

During the initiation of mitochondria-dependent apoptosis, Bim, a crucial upstream molecule in this process, acts as a powerful inducer of apoptosis by triggering mitochondrial disruption and decreasing mitochondrial membrane potentials [20, 21]. Increased expression of Bim by pro-apoptotic agents induces the activation of Bax and attenuates the anti-apoptotic effects of Bcl-2 [22]. These pro-apoptotic changes subsequently lead to decreased MMP and increased mitochondrial permeability, which promotes the release of cytochrome $\mathrm{C}$ from mitochondria into the cytoplasm. Cytochrome $\mathrm{C}$ in the cytoplasm, in turn, prompts the activation of caspase cascades and triggers cell apoptosis [23, 24]. To explore the role of KDM6B in apoptosis, we analyzed the expression of Bim and changes in MMP. Our results showed that KDM6B overexpression significantly increased the expression of Bim and decreased MMP, resulting in a disruption to mitochondrial integrity. Conversely, knocking down expression of KDM6B inhibits the expression of Bim and caspase-9 activation. These results suggest that KDM6B initiates mitochondria-dependent apoptosis.

The transcription factor FOXO1 regulates growth arrest and apoptosis by activating various cellular processes [25]. Cell homeostasis is normally maintained through a dynamic balance involving the constant shuttling of FOXO1 between the nucleus and cytoplasm [26, 27]. FOXO1 induces cell apoptosis following its translocation into the nucleus, where it increases the expression of several genes involved in the apoptotic pathway. Phosphorylation of FOXO1 results in its inactivation and exclusion from the nucleus [28, 29]. It has been reported that FOXO1 directly binds to specific response elements in the Bim promoter to modulate Bim gene expression [30, 31]. In this study, we found that KDM6B directly binds FOXO1 and induces its nuclear accumulation. This observation is in accordance with our results showing that overexpression of KDM6B up-regulates the expression of Bim and initiates mitochondria-dependent apoptosis. Moreover, we also confirmed that the apoptotic effects of KDM6B are mediated by FOX01. These results indicate that KDM6B induces apoptosis by promoting the nuclear translocation of FOXO1.

Invasion-metastasis cascades are an important biological characteristic of malignant tumors and a leading cause of death in patients. Because invasion and migration lead to the spread of cancer cells to anatomically distant organs, these processes greatly exacerbate the difficulty of operations and increase the risk of tumor recurrence in other tissues [32, 33]. To explore the role of KDM6B in the progression of NSCLC, we studied the effects of KDM6B on the invasion-metastasis cascade in A549 and H460 cells and found that KDM6B overexpression reduced migration and invasion and mitigated cancer aggressiveness in NSCLC. The inhibitory effects of KDM6B on invasive behavior were attenuated by siFOX01, suggesting that KDM6B regulates the invasion-metastasis cascade by interacting with FOXO1 in NSCLC. Although we have demonstrated that KDM6B inhibits apoptosis and metastasis in NSCLC cells via an interaction with FOXO1, other parallel signaling pathways may also be 


\section{Cellular Physiology Cell Physiol Biochem 2015;37:201-213 \begin{tabular}{c|c}
\hline DOI: 10.1159/000430345 & (c) 2015 S. Karger AG, Basel
\end{tabular} \begin{tabular}{l|l} 
and Biochemistry Published online: August 20, 2015 & www.karger.com/cpb \\
\hline
\end{tabular}}

Ma et al.: KDM6B Elicits Apoptosis by FOXO1 in NSCLCS

involved in the KDM6B modulated inhibition of apoptosis and metastasis, and this will need to be further studied.

In summary, we have shown that FOX01 mediates the inhibitory effects of KDM6B on cell apoptosis and metastasis, suggesting that KDM6B acts as a tumor suppressor in NSCLC. Therefore, KDM6B may be a novel potential target for the development of strategies used to treat NSCLC.

\section{Acknowledgements}

This work was supported by the grants from the State Key Laboratory of Oncogenes and Related Genes (No. 90-14-07) and the China Postdoctoral Science Foundation (No. 2014M551423).

\section{Disclosure Statement}

None.

\section{References}

1 Siegel R, Naishadham D, Jemal A: Cancer statistics, 2012. CA Cancer J Clin 2012;62:10-29.

2 Wang J, Yang B, Han L, Li X, Tao H, Zhang S, Hu Y: Demethylation of miR-9-3 and miR-193a genes suppresses proliferation and promotes apoptosis in non-small cell lung cancer cell lines. Cell Physiol Biochem 2013;32:1707-1719.

3 Herbst RS, Heymach JV, Lippman SM: Lung cancer. N Engl J Med 2008;359:1367-1380.

4 Zhang L, Qian J, Qiang Y, Huang H, Wang C, Li D, Xu B: Down-regulation of miR-4500 promoted non-small cell lung cancer growth. Cell Physiol Biochem 2014;34:1166-1174.

5 Wu N, Zhang C, Bai C, Han YP, Li Q: MiR-4782-3p inhibited non-small cell lung cancer growth via USP14. Cell Physiol Biochem 2014;33:457-467.

6 Evan GI, Vousden KH: Proliferation, cell cycle and apoptosis in cancer. Nature 2001;411:342-348.

7 Kouzarides T: Chromatin modifications and their function. Cell 2007;128:693-705.

8 Peterson CL, Laniel MA: Histones and histone modifications. Curr Biol 2004;14:R546-551.

9 Kim JH, Sharma A, Dhar SS, Lee SH, Gu B, Chan CH, Lin HK, Lee MG: UTX and MLL4 coordinately regulate transcriptional programs for cell proliferation and invasiveness in breast cancer cells. Cancer Res 2014;74:1705-1717.

10 Teng YC, Lee CF, Li YS, Chen YR, Hsiao PW, Chan MY, Lin FM, Huang HD, Chen YT, Jeng YM, Hsu CH, Yan Q, Tsai MD, Juan LJ: Histone demethylase RBP2 promotes lung tumorigenesis and cancer metastasis. Cancer Res 2013;73:4711-4721.

11 Hoffmann I, Roatsch M, Schmitt ML, Carlino L, Pippel M, Sippl W, Jung M: The role of histone demethylases in cancer therapy. Mol Oncol 2012;6:683-703.

12 Yamamoto K, Tateishi K, Kudo Y, Sato T, Yamamoto S, Miyabayashi K, Matsusaka K, Asaoka Y, Ijichi H, Hirata Y, Otsuka M, Nakai Y, Isayama H, Ikenoue T, Kurokawa M, Fukayama M, Kokudo N, Omata M, Koike K: Loss of histone demethylase KDM6B enhances aggressiveness of pancreatic cancer through downregulation of C/ EBP $\alpha$. Carcinogenesis 2014;35:2404-2414.

13 Pereira F, Barbáchano A, Silva J, Bonilla F, Campbell MJ, Muñoz A, Larriba MJ: KDM6B/JMJD3 histone demethylase is induced by vitamin $\mathrm{D}$ and modulates its effects in colon cancer cells. Hum Mol Genet 2011;20:4655-4665.

14 Anderton JA, Bose S, Vockerodt M, Vrzalikova K, Wei W, Kuo M, Helin K, Christensen J, Rowe M, Murray PG, Woodman CB: The H3K27me3 demethylase, KDM6B, is induced by Epstein-Barr virus and over-expressed in Hodgkin's Lymphoma. Oncogene 2011;30:2037-2043.

15 Ramadoss S, Chen X, Wang CY: Histone demethylase KDM6B promotes epithelial-mesenchymal transition. J Biol Chem 2012;287:44508-44517. 


\section{Cellular Physiology Cell Physiol Biochem 2015;37:201-213 \begin{tabular}{c|c}
\hline DOI: 10.1159/000430345 & (c) 2015 S. Karger AG, Basel
\end{tabular} \begin{tabular}{l|l} 
and Biochemistry Published online: August 20, 2015 & www.karger.com/cpb \\
\hline
\end{tabular}}

Ma et al.: KDM6B Elicits Apoptosis by FOXO1 in NSCLCS

16 Shen Y, Guo X, Wang Y, Qiu W, Chang Y, Zhang A, Duan X: Expression and significance of histone H3K27 demethylases in renal cell carcinoma. BMC Cancer 2012;12:470.

17 Estarás C, Akizu N, García A, Beltrán S, de la Cruz X, Martínez-Balbás MA: Genome-wide analysis reveals that Smad3 and JMJD3 HDM co-activate the neural developmental program. Development 2012;139:26812691.

18 Zhao W, Li Q, Ayers S, Gu Y, Shi Z, Zhu Q, Chen Y, Wang HY, Wang RF: Jmjd3 inhibits reprogramming by upregulating expression of INK4a/Arf and targeting PHF20 for ubiquitination. Cell 2013;152:1037-1050.

19 Dahle Ø, Kumar A, Kuehn MR: Nodal signaling recruits the histone demethylase Jmjd3 to counteract polycomb-mediated repression at target genes. Sci Signal 2010;3:ra48.

20 Puthalakath H, Huang DC, O'Reilly LA, King SM, Strasser A: The proapoptotic activity of the Bcl-2 family member Bim is regulated by interaction with the dynein motor complex. Mol Cell 1999;3:287-296.

21 Reed JC: Bcl-2 family proteins. Oncogene 1998;17:3225-3236.

22 Shukla S, Rizvi F, Raisuddin S, Kakkar P: FoxO proteins' nuclear retention and BH3-only protein Bim induction evoke mitochondrial dysfunction-mediated apoptosis in berberine-treated HepG2 cells. Free Radic Biol Med 2014;76:185-199.

23 Jiang X, Jiang H, Shen Z, Wang X: Activation of mitochondrial protease OMA1 by Bax and Bak promotes cytochrome c release during apoptosis. Proc Natl Acad Sci U S A 2014;111:14782-14787.

24 Li P, Nijhawan D, Budihardjo I, Srinivasula SM, Ahmad M, Alnemri ES, Wang X: Cytochrome c and dATP-dependent formation of Apaf-1/caspase-9 complex initiates an apoptotic protease cascade. Cell 1997;91:479-489.

25 Fu Z, Tindall DJ: FOXOs, cancer and regulation of apoptosis. Oncogene 2008;27:2312-2319.

26 Brunet A, Bonni A, Zigmond MJ, Lin MZ, Juo P, Hu LS, Anderson MJ, Arden KC, Blenis J, Greenberg ME: Akt promotes cell survival by phosphorylating and inhibiting a Forkhead transcription factor. Cell 1999;96:857-868.

27 Biggs WH 3rd, Meisenhelder J, Hunter T, Cavenee WK, Arden KC: Protein kinase B/Akt-mediated phosphorylation promotes nuclear exclusion of the winged helix transcription factor FKHR1. Proc Natl Acad Sci U S A 1999;96:7421-7426.

28 Nakae J, Barr V, Accili D: Differential regulation of gene expression by insulin and IGF-1 receptors correlates with phosphorylation of a single amino acid residue in the forkhead transcription factor FKHR. EMBO J 2000;19:989-996.

29 Alikhani M, Alikhani Z, Graves DT: FOXO1 functions as a master switch that regulates gene expression necessary for tumor necrosis factor-induced fibroblast apoptosis. J Biol Chem 2005;280:12096-12102.

30 Shore AM, White PC, Hui RC, Essafi A, Lam EW, Rowe M, Brennan P: Epstein-Barr virus represses the Fox01 transcription factor through latent membrane protein 1 and latent membrane protein 2A. J Virol 2006;80:11191-11199.

31 McLoughlin TJ, Smith SM, DeLong AD, Wang H, Unterman TG, Esser KA: FoxO1 induces apoptosis in skeletal myotubes in a DNA-binding-dependent manner. Am J Physiol Cell Physiol 2009;297:C548-555.

32 Chaffer CL, Weinberg RA: A perspective on cancer cell metastasis. Science 2011;331:1559-1564.

33 Sulzmaier FJ, Ramos JW: RSK isoforms in cancer cell invasion and metastasis. Cancer Res 2013;73:60996105. 